\title{
Airglow remote sensing of the seasonal variation of the Martian upper atmosphere: MAVEN limb observations and model comparison
}

\author{
L. Gkouvelis $^{\text {a,*, J.-C. Gérard }}{ }^{\mathrm{a}}$, B. Ritter ${ }^{\mathrm{a}, 1}$, B. Hubert ${ }^{\mathrm{a}}$, N.M. Schneider ${ }^{\mathrm{b}}$, S.K. Jain ${ }^{\mathrm{b}}$ \\ ${ }^{a}$ LPAP, STAR Institute, Université de Liège, Belgium \\ ${ }^{\mathrm{b}}$ LASP, University of Colorado, USA
}

\section{A R T I C L E I N F O}

\section{Keywords:}

Mars

Airglow

MAVEN

IUVS

Seasonal variation

\begin{abstract}
A B S T R A C T
We apply a new technique to remotely sense the seasonal altitude variation of the $0.39 \mu$ bar isobar level in this region. We present results from observations of airglow limb profiles collected during two Martian years with the Imaging UltraViolet Spectrograph (IUVS) onboard MAVEN. IUVS has collected tens of thousands of Martian airglow limb profiles since the Fall of 2014. We analyze the global dayglow dataset of the $297.2 \mathrm{~nm}$ emission corresponding to the $\left.\mathrm{O}^{3} \mathrm{P}^{1}{ }^{1} \mathrm{~S}\right)$ forbidden transition. The vertical profile of this unique emission shows two peaks: one around $120 \mathrm{~km}$ and a second one near $80 \mathrm{~km}$. We use both peaks to trace the altitude changes of the isobars in this atmospheric region. We map and compare them with the seasonal variations of the $\mathrm{O}\left({ }^{1} \mathrm{~S}\right)$ with $\mathrm{CO}_{2}^{+} \mathrm{UV}$ doublet peak. We find that the altitude of the $0.39 \mu \mathrm{bar}$ level varies by up to $20 \mathrm{~km}$. We show that the dayglow model overpredicts the lower peak altitude by $2-5 \mathrm{~km}$ from the observations while it underpredicts the upper peak altitude with the same offset. The best agreement near $80 \mathrm{~km}$ is obtained by decreasing the $\mathrm{CO}_{2}$ density by the Mars Climate Database (MCD) by about $40 \%$.
\end{abstract}

\section{Introduction}

Mars, similar to the Earth, has four seasons due to the $25^{\circ}$ inclination of its rotation axis. These seasonal changes are followed by a $\mathrm{CO}_{2}$ trapping in the ice phase, in both polar regions of the planet, leading to significant seasonal variations of the total pressure of the Martian surface atmosphere. The eccentricity of the Martian orbit and the concentration of the dust storms in southern summer lead to an asymmetry in the sublimation of $\mathrm{CO}_{2}$ in the polar caps. This explains why the ground pressure is largest around solar longitudes close to 270 degrees (Hess et al., 1980). These pressure variations have been monitored in the past by landers and rovers. The Viking 1 and 2 landers (Hanson et al., 1977; Nier and McElroy, 1977) have measured up to $\sim 30 \%$ changes of the ground pressure (Hess et al., 1980).

Seasonal neutral density variations between 70 and $130 \mathrm{~km}$ have also been shown using solar occultation techniques (Forget et al., 2009; Gröller et al., 2018). Ionospheric seasonal variations have been studied in terms of in-situ measurements, solar and stellar occultation techniques by Withers and Pratt (2013). In-situ measurements have been collected from deep-dip measurements (MAVEN, Viking 1 \& 2 measurements). In this work, we apply a new remote sensing technique to trace the variation of the pressure in the 70-95 km region and present thereby a useful tool to complete these types of studies.
The lower thermosphere is a region that links the lower atmosphere to the upper-atmosphere and new mechanisms start to play a role in driving physics of the atmospheric composition and dynamics. Ultraviolet (UV) radiation plays an increasingly important role in the heating of the atmosphere, so that the driving physics and chemistry composition and the dynamics will differ of the lower atmospheric layers.

To follow the altitude variation of the isobars, we use the vertical emission profile of the OI $297.2 \mathrm{~nm}$ line in the mid-UV (MUV) spectral region. The $297.2 \mathrm{~nm}$ emission originates from the ${ }^{1} S$ metastable state of $\mathrm{O}$ atoms in the fundamental electron configuration. It corresponds to the $\mathrm{OI}^{3} \mathrm{P}-{ }^{1} S$ singlet magnetic dipole transition with transition probability of $7.54 \times 10^{-2} \mathrm{~s}^{-1}$. It shares this upper energy state with the transition at $557.7 \mathrm{~nm}$, the so-called auroral green line with a ratio, $\mathrm{R}=\mathrm{A}_{555.7} / \mathrm{A}_{297.2}$, equal to 15.5 (Chantler et al., 2013; NIST: Kramida et al., 2018) .

It was first detected in the Martian airglow by the ultraviolet spectrometers during the Mariner 6 and 7 flybys (Barth et al., 1971; Stewart, 1972) and later observed from the Mariner 9 orbiter (Barth et al., 1972; Stewart et al., 1972). This emission was later observed at the limb with the Spectroscopy for the Investigation of the Characteristics of the Atmosphere of Mars (SPICAM) UV spectrometer (Bertaux et al., 2006) on board Mars Express. Leblanc et al. (2006) detected an emission peak

\footnotetext{
* Corresponding author.

E-mail address: 1.gkouvelis@uliege.be (L. Gkouvelis).

1 Now at Royal observatory of Belgium, Brussels, Belgium.
} 
around $120 \mathrm{~km}$. Observations at altitudes less than $100 \mathrm{~km}$ were not possible with SPICAM at this wavelength due to the presence of strong solar scattered light.

A first model of the production of this emission was presented by Fox and Dalgarno (1979) who predicted the presence of a double peak with the strongest emission lying around $80 \mathrm{~km}$. This lower peak is produced by solar Lyman- $\alpha$ radiation at $121.6 \mathrm{~nm}$ that photodissociates $\mathrm{CO}_{2}$ leaving a fraction of the $\mathrm{O}$ atoms in the ${ }^{1} \mathrm{~S}$ state. The first observations of the lower peak emission were provided by the Imaging UltraViolet Spectrograph (IUVS) (McClintock et al., 2015) on board the Mars Atmosphere and Volatile EvolutioN (MAVEN) orbiter (Jain et al., 2015). Model-observations comparisons for different seasons were described by Gkouvelis et al. (2018) (hereafter referred to as G18) who showed that the $\mathrm{O}\left({ }^{1} \mathrm{~S}\right)$ emission is an ideal tracer of the isobar variations in the 70-95 $\mathrm{km}$ region. This is possible since $\mathrm{CO}_{2}$ is the only significant absorber of solar Lyman- $\alpha$ flux and the $297.2 \mathrm{~nm}$ peak altitude only depends on the overlying column of carbon dioxide. This particularity is explained by a local minimum in the $\mathrm{CO}_{2}$ absorption cross section near this wavelength (Yoshino et al., 1996; Venot et al., 2018) and the intensity dominance by Lyman- $\alpha$ by two orders of magnitude in the far UV spectral region. Lyman- $\alpha$ penetrates therefore deep into the atmosphere and creates a lower peak around $80 \mathrm{~km}$. The result is that the vertical emission profile is controlled by a single wavelength and a single absorber. That makes this emission unique in the Martian atmosphere and it may be analytically expressed as a Chapman layer (Gkouvelis et al. 2018). The optical depth at Lyman- $\alpha$ reaches unity at the peak of the lower vertical profile, allowing to know the exact slant column density of $\mathrm{CO}_{2}$ and thus a pressure determination of this altitude. We can thus detect and follow the variations of the lower emission peak across the different seasons in the Martian year to study the altitude variation of the isobar.

We present the analysis of four years (two Martian years) of IUVS/MAVEN limb scans and compare simultaneous measurements of the $\left.\mathrm{O}^{1} \mathrm{~S}\right)$ and $\mathrm{CO}_{2}^{+} \mathrm{UV}$ doublet emissions. We also compare the observed seasonal changes with simulations based on the MCD neutral density model for the same periods. Therefore, we are able to observe altitude variations of two atmospheric layers difficult to monitor in situ.

\section{Observations}

MAVEN entered in a Martian orbit on the Fall of 2014 Jakosky (2017). One of the eight scientific instruments on board is the Imaging Ultraviolet Spectrometer (IUVS). It supports two spectroscopic modes, using two separate gratings that provide the required resolving powers. One of the two operates near normal incidence and covers the 110$340 \mathrm{~nm}$ range with a resolving power $\sim 250$. In this work we analyze data collected in the atmospheric limb scan observation mode. During each orbit, the detector performs 12 vertical scans with a mirror rotating between 21 angular positions. In the data reduction procedure, the vertical data points are separated into bins of $5 \mathrm{~km}$ for the purpose of the homogeneity of the data. The observations are available at the NASA Planetary Data System (PDS) at four levels of processing. We use level 1C, version 13, IUVS data available from the PDS public archives. The orbit range spans from the 108 to 7490, which corresponds to October 18, 2014 till August 4, 2018. For this study, we apply a correction factor of 0.69 to the $\mathrm{O} I 297.2 \mathrm{~nm}$ absolute intensities, following the calibration revision made in November 2018.

For the purpose of this work we also use data from the Solar Extreme Ultraviolet Monitor (EUVM) experiment (Eparvier et al., 2015) on board MAVEN in order to model the $\mathrm{O}\left({ }^{1} \mathrm{~S}\right)$ vertical production rate using solar radiation input appropriate to concurrent dayglow observations. We use the daily averaged modeled solar spectra in the range of 1-160 nm (Thiemann et al., 2016). These spectra are modeled based on three channel observations: Lyman- $\alpha, 17-22 \mathrm{~nm}$ and $0-7 \mathrm{~nm}$. The reason why the OI $297.2 \mathrm{~nm}$ lower emission peak was only detected in late 2014, with IUVS, is mainly because the solar stray light was

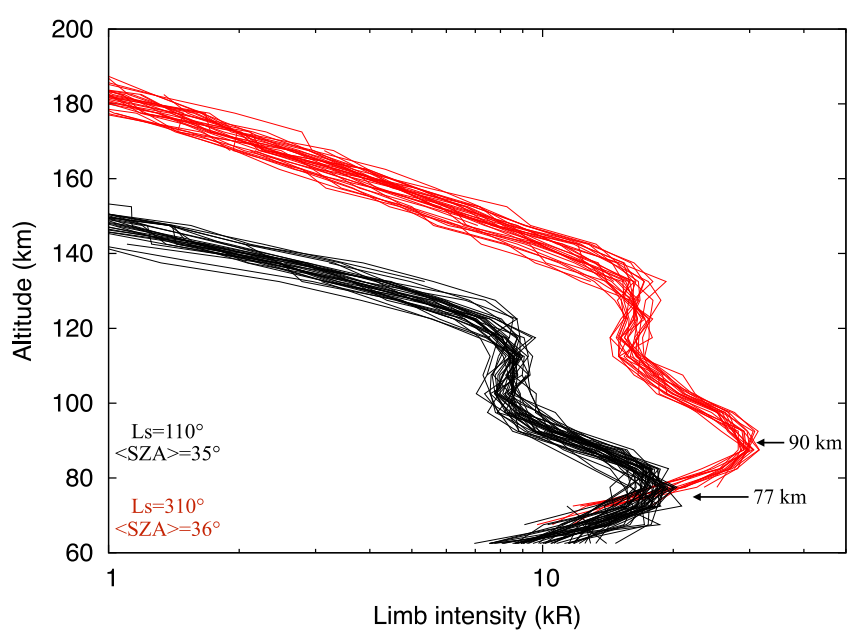

Fig. 1. IUVS observations of two sub-groups of limb scan at different season, but nearly equal solar zenith angle.

avoided with improved instrument baffling. A sophisticated technique was used to extract what is left from the solar continuum from our spectra, as was shown in Fig. 1 by G18 and described by Stevens et al. (2015). The shape of this solar scattered light component is an excellent estimate of the scattered light embedded in the airglow signal and efficiently removes the solar stray light contribution at these altitudes.

In order to rely on statistically significant limb profiles, we base our analysis on mean limb profiles. These mean profiles are created by selecting limb scans meeting conditions of parameter ranges for each individual case. The observations have been sorted according to $L_{s}$, latitude, solar zenith angle (SZA), Martian year and local time. The bins are $10^{\circ} \times 10^{\circ}$ in $L_{s}$ and latitude, $0^{\circ}-30^{\circ}, 30^{\circ}-45^{\circ}, 45^{\circ}-60^{\circ}, 60^{\circ}-70^{\circ}$ and $70^{\circ}-75^{\circ}$ bins in solar zenith angle. Local time is only used as an indicator to avoid mixing morning with afternoon data. The amplitude of the above ranges was fixed in order to automatically separate the IUVS database in sub-groups but the typical mean profile has also a mean value of those quantities with a standard deviation usually lower than the initial range limits. Fig. 1 shows an example of two groups of individual vertical scans belonging to two different Martian seasons. Only the upper peak altitude measurements, with a SZA less than $45^{\circ}$ are used, for reasons discussed in Section 4.

In this work, the remote sensing methodology and results concentrate on the lower peak, thus from now all discussions refer to it. We have analyzed 6150 IUVS limb scans to create 246 dayglow $\left(\right.$ SZA $\left.<75^{\circ}\right)$ mean profiles containing at least 25 scans each. In this database 77 have SZA $<45^{\circ}$ that were used to determine the altitude of the upper peak. We can reach a one-kilometer resolution in the altitude of the tangent point, based on a quadratic fit around the peak, as was shown in G18. The intensity standard deviation, as it is calculated from the sub-grouping per pixel, of the mean profile peak intensity is typically in the order of $1.5 \mathrm{kR}$ and reaches $2.5 \mathrm{kR}$ at the peak.

\section{Methodology}

The method used in this work, was described in G18, to analyze the characteristics of the newly discovered OI $297.2 \mathrm{~nm}$ lower emission peak. It is based on the simple physical mechanism that is described by the Chapman layer theory of one monochromatic wavelength interacting with one absorber. It is then possible to analytically calculate where the optical depth of Lyman- $\alpha$ reaches unity in $\mathrm{CO}_{2}$ and determine the $\mathrm{CO}_{2}$ slant column density $\left(N_{0}\right)$, depending on the solar zenith angle (SZA). The constant slant column density for unit optical depth is $1.5 \times 10^{19} \mathrm{~cm}^{-2}$. Integration of the production rate along the line of sight affects the altitude of the peak of the limb profile and lowers it by $5 \pm 0.5$ 


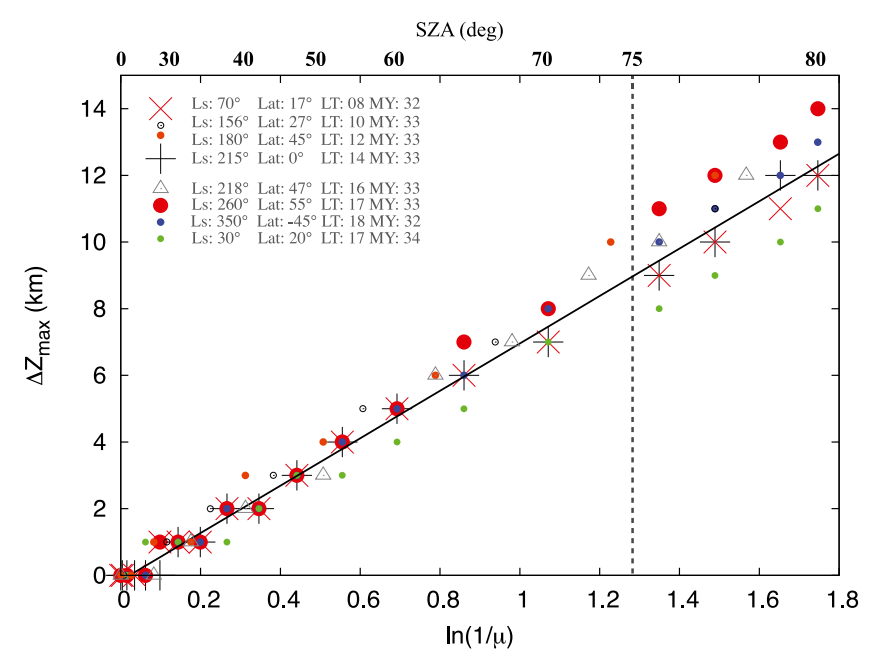

Fig. 2. The peak altitude difference $\Delta Z_{\max }$ between $Z_{\max }^{0}$, for $S Z A=0^{\circ}$ and $Z_{\max }^{\theta}$ as a function of $\ln (1 / \cos (\theta))$ for different neutral atmospheres extracted from the MCD, given by different symbols. Next to each symbol we give the generating parameters of each neutral atmosphere. The black line is the linear fit and the black dashed line is the limit we have set at $S Z A=75^{\circ}$. We conclude that we can transform each lower peak altitude to its reference zero angle by using the mean SZA value of the observations at the lower peak.

$\mathrm{km}$, independently of the SZA. This shift of the peak also increases the slant column density to the constant value of $N_{0}=(2.2 \pm 0.2) \times 10^{19} \mathrm{~cm}^{-2}$. For a vertical solar flux incident and since the $\mathrm{CO}_{2}$ makes $96 \%$ of the atmospheric composition, we can calculate the pressure $P_{0}$ at the emission peak with a vertical uncertainty of about $1 \mathrm{~km}$ leading to a value $P_{0}=(0.39 \pm 0.03) \mu$ bar or $(3.9 \pm 0.3) \times 10^{-2} \mathrm{~Pa}$.

The $P_{0}$ value calculated in the previous section was for the vertical column density and applies with a good approximation for SZA up to $45^{\circ}$. However, the IUVS database includes dayglow data at all solar zenith angles, most of them for SZA higher than $45^{\circ}$. The constant quantity controlling the location of the emission peak is the slant $\mathrm{CO}_{2}$ column density. The pressure at any level only depends on the overlying vertical column density so that the dependence is proportional to $\cos (\theta)$ in good approximation for angles less than $\sim 75^{\circ}$, where $\theta$ is the solar zenith angle. It is convenient to work at a constant pressure level and transform the slant column at solar zenith angle $\theta$ into an equivalent vertical column for all observations in the database. This can be done in this case since we know analytically that the peak altitude $\left(Z_{\max }\right)$ of a Chapman layer behaves:

$Z_{\max } \sim \ln (1 / \mu)$

where $Z_{\max }$ is the peak altitude of the production rate and $\mu \equiv \cos (\theta)$. We have confirmed this result by experimenting with our models and examining the dependence between the solar zenith angle and the corresponding changes in the integrated peak altitude $\left(\Delta Z_{\max }\right)$. We generate various neutral atmospheres with different parameters as can be seen in Fig. 2 and we simulate each individual case for solar zenith angles ranging from $0^{\circ}$ up to $80^{\circ}$. In Fig. 2, we plot the changes in the altitude of the peak, relative to $Z_{\max }$ for $S Z A=0^{\circ}$ for each case as function of $\ln (1 / \mu)$. The correlation coefficient of the fit is 0.98 with a constant of $7.1 \mathrm{~km}$. The standard deviation of the fit grows proportionally to the angle. At $75^{\circ}$ it reaches a $1-\mathrm{km}$ uncertainty. In order to be consistent with the model and our methodology that was described by G18 we choose to restrict our analysis to data with SZA $<75^{\circ}$. The upper peak is produced by a range of EUV solar wavelengths so that it cannot be treated analytically as a Chapman function. For this reason we choose to extract the peak altitude only for observations with SZA $<45^{\circ}$. The upper peak data were analyzed following the methodology described by Gérard et al..

\section{Results}

We have analyzed the whole IUVS database and created 246 dayglow (SZA $<75^{\circ}$ ) mean profiles with transformed lower peak altitudes which represent the reference pressure level of $P_{0}=(3.9 \pm 0.3) \pm 10^{-2}$ $\mathrm{Pa}\left(0.39 \times 10^{-6}\right.$ bar). In Fig. 3(a), we plot the altitude of $P_{0}$ for the first three Martian years when IUVS was operated. The mean value of the solar Longitude $\left(L_{s}\right)$ is shown versus mean topological latitude (Lat) for each lower peak vertical scan group. We plot the pixels in terms of $L_{s}$ and latitude so that the bins have a $10^{\circ} \times 10^{\circ}$ size. Fig. 3(b) shows the altitude of the same reference pressure $P_{0}$ extracted from the MCD. For each data bin we derived mean values that are used as generic parameters for the Mars Climate Database (MCD) and we determine the altitude of the $P_{0}$ pressure level.

We use the 5.3 version of the MCD (Forget et al., 1999; GonzálezGalindo et al., 2005; Millour et al., 2015) which includes the dust scenarios for Martian years 32 and 33 (Montabone et al., 2015). For Martian year 34, we use the scenario of the MCD solar minimum climatology corresponding to the solar activity of this period. We show the simulated results in the same format as the observations for the same dates, latitude and longitudes. The comparison of the two panels will be discussed in the next section. Finally, in Fig. 4 we present a combination of results with no latitudinal distinction. We show the two $\mathrm{O}\left({ }^{1} \mathrm{~S}\right)$ peak altitudes and the $\mathrm{CO}_{2}^{+} \mathrm{UVD}$ as was presented by Gérard et al. (2019) for comparison purposes. The bottom panel of the figure presents the observations of grouped pixels in green, purple and blue for Martian years 32, 33 and 34 respectively. The filled circles represent the OI $297.2 \mathrm{~nm}$ lower peak, squares the OI $297.2 \mathrm{~nm}$ upper peak and triangles correspond to the $\mathrm{CO}_{2}^{+} \mathrm{UVD}$ emission. In this plot, the $297.2 \mathrm{~nm}$ upper peak plot has been shifted down by $15 \mathrm{~km}$ for clarity reasons. We overplot with small dots, black and orange, the 1-D simulations using MCD neutral input atmospheres for the same conditions as the observations. The small red dots follow the $\mathrm{P}_{0}$ pressure as was explained in the previous section. The top panel of the figure shows the scaling factor (in percent) that was applied to the $\mathrm{CO}_{2}$ density profile from the MCD to obtain a perfect match between the observed and the simulated peak.

In Fig. 3 we plot separately each Martian year and a clear seasonal pattern is seen on the change of the isobar altitude. This pattern is predicted by the LMD-GCM simulations. It is also represented in Fig. 4, where we merge all Martian years and directly compare with the model. The seasonal variation of the upper peak shows the same trend as the lower peak. They are separated by a distance of $34 \pm 2 \mathrm{~km}$ across the whole observation period. The same pattern was shown by Gérard et al. (2019) for $\mathrm{CO}_{2}^{+}$UVD emission. This similarity is expected since both emissions directly depend on the $\mathrm{CO}_{2}$ photodissociation. In terms of seasonal trend, observations and model totally agree in spite of a systematic difference of $2-5 \mathrm{~km}$ during the first part of the Martian year. However the offset almost completely disappears for the lower peak during dust storm periods ( $L s>180^{\circ}$ ). In contrast, the dust period is when the maximum discrepancy is observed for the two emissions at higher altitudes. Outside the dust periods, we have performed onedimensional airglow model calculations to scale the modeled values from the MCD. We conclude that if the $\mathrm{CO}_{2}$ density profile is multiplied by a factor of about 0.6 , a nearly perfect match is obtained, in agreement with earlier work by Gkouvelis et al. (2018). Inversely, if we scale the MCD density values to match the observations, for $0^{\circ}<\mathrm{Ls}$ $<180^{\circ}$, we are left with a discrepancy during the dust storm periods. In these conditions, the model underestimates the peak altitude by 2 to 5 $\mathrm{km}$. At the higher altitudes we find that to obtain a good match we need to scale the $\mathrm{CO}_{2}$ density by 1.3 outside of the dusty season. However, the $\mathrm{CO}_{2}^{+} \mathrm{UVD}$ emission is not as well reproduced, especially Ls between $220^{\circ}-360^{\circ}$. This suggests that during this season, the propagation of the perturbation by the dust storm between the two layers is not properly modeled. The altitude of the $297.2 \mathrm{~nm}$ emissions peaks varies by $\sim 20$ $\mathrm{km}$ during a Martian year including both the seasonal variation due to the varying distance from the sun and the additional perturbation resulting from the dust storm. 


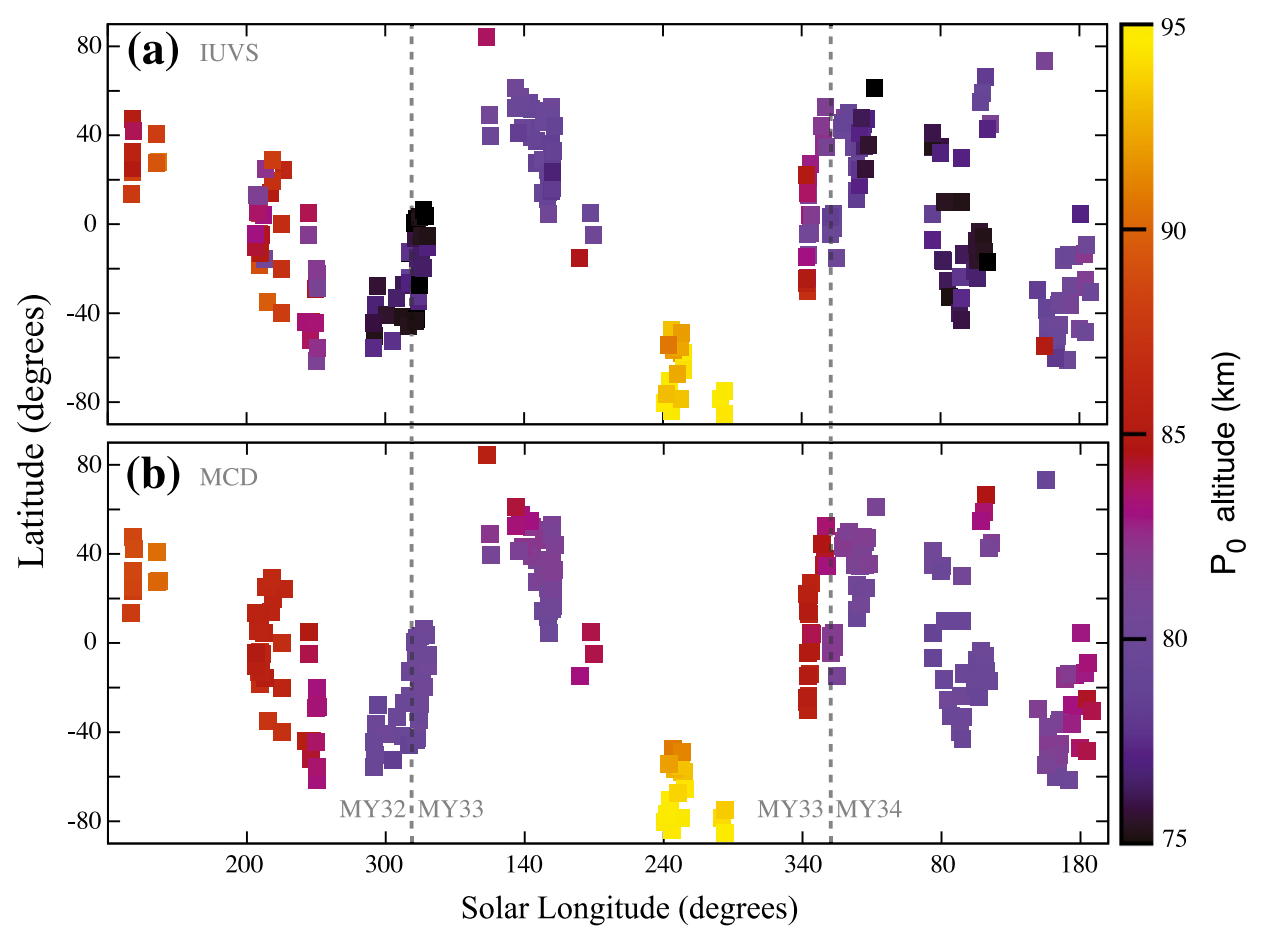

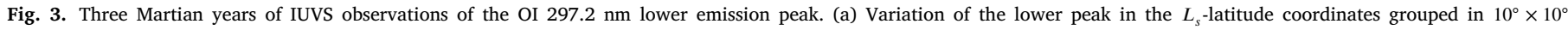

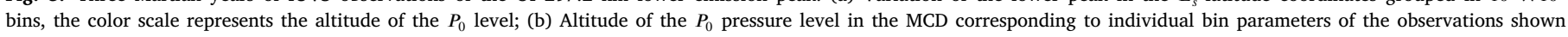
in (a). (For interpretation of the references to color in this figure legend, the reader is referred to the web version of this article.)

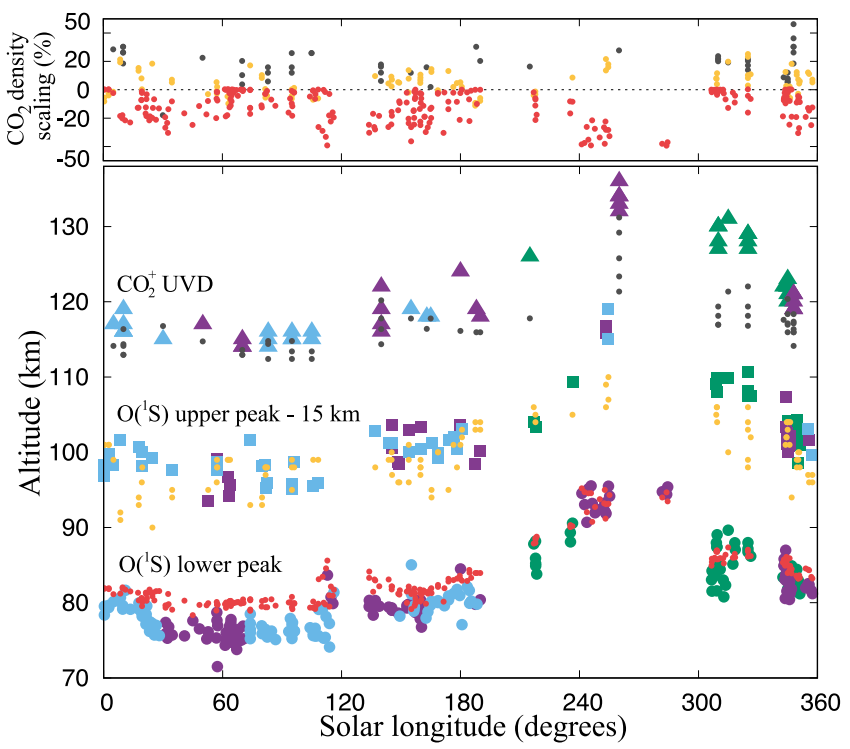

Fig. 4. Observed and modeled comparisons of the peak altitudes of the $O\left({ }^{1} S\right)$ and $\mathrm{CO}_{2}^{+}$UVD emissions across all Martian seasons. Bottom: The observations are shown in green, purple and blue for Martian years 32, 33 and 34. The filled circles represent the OI $297.2 \mathrm{~nm}$ lower peak, squares the OI $297.2 \mathrm{~nm}$ upper peak and triangles correspond to the $\mathrm{CO}_{2}^{+}$UVD emission. The $297.2 \mathrm{~nm}$ upper peak plot has been shifted down by $15 \mathrm{~km}$ for clarity. Small, black and orange dots, correspond to 1-D simulations using MCD neutral input atmospheres for the same conditions as the observations. The top panel shows the scaling factor (in percent) that was applied to the $\mathrm{MCD} \mathrm{CO}_{2}$ density profile in order to match the simulated and the observed peaks.

\section{Discussion}

We present the yearly isobar variation in the altitude range of 70$95 \mathrm{~km}$ in Figs. 3 and 4. In the upper panel of Fig. 4, we clearly see the difference in the $\mathrm{CO}_{2}$ density discrepancy between the observations and the model for the two atmospheric levels. For the lower altitudes, $94 \%$ of the sample needs to be scaled with a factor less than 1 , while a scaling greater than unity is required for $98 \%$ of the data point corresponding to the $297.2 \mathrm{~nm}$ upper layer and $70 \%$ for $\mathrm{CO}_{2}^{+} \mathrm{UVD}$. The results of the $\mathrm{O}\left({ }^{1} \mathrm{~S}\right)$ upper layer are in agreement with the conclusion by González-Galindo et al. (2018) and Gérard et al. (2019) for the altitude range $110-130 \mathrm{~km}$. The same pattern was found at various altitudes, in terms of $\mathrm{CO}_{2}$ density as a function of season, by Forget et al. (2009), based on SPICAM stellar occultation measurements. However, stellar occultations measurements were mostly limited to the nightside.

The difference in scaling factor (1.3 compared to 0.6$)$ needed to reconcile the modeled peak altitude of the three emissions with the observations suggests that the MCD $\mathrm{CO}_{2}$ vertical distribution in the upper atmosphere may be improved. This distribution depends, in turn, on the vertical temperature distribution. The temperature profile in this region is controlled by the balance between solar and dynamical heating, radiative cooling and heat conduction González-Galindo et al. (2005). It generally shows a minimum between 100 and $120 \mathrm{~km}$ (Gröller et al., 2018). The measured temperature difference between the two levels appears quite variable with season, SZA and latitude. Our results suggest that the temperature gradient between the levels of the $297.2 \mathrm{~nm}$ lower and the upper peaks (as well as that of $\mathrm{CO}_{2}^{+}$ UVD) is not in agreement with that predicted by the MCD. The model overestimates or underestimates the temperature vertical gradient. We also note that the altitude of the homopause varies from $130 \mathrm{~km}$ to $80 \mathrm{~km}$ as a function of SZA and season Jakosky (2017). Therefore, the $\mathrm{CO}_{2}$ mixing ratio is also variable at $120 \mathrm{~km}$, which may also explain the discrepancy between the different pressure levels.

The $\mathrm{P}_{0}$ detection technique is complementary to the stellar and solar occultation remote sensing methods. The altitude region considered in this study includes the mesopause ( 80 to $100 \mathrm{~km}$ ) and connects the lower with the upper atmosphere. This is the region where heating by solar UV radiation starts to become important. Our method provides the possibility of remote sensing this region, which is otherwise very difficult to probe by in situ methods. 
MAVEN's investigation of the Martian atmosphere is ongoing and will possibly continue for several years to come, enabling us to enrich our results and obtain even better statistics. It is possible to apply the same technique to individual limb scans in order to improve the time resolution and track fast atmospheric changes. The emission at $297.2 \mathrm{~nm}$ is also sensitive to tides and gravity waves that perturb this atmospheric layer. This calculation is for mean profiles that average out short-time fluctuations. Future observations can use this sensitive method in order to observe and track continuously isobar altitude variations related to short-term tide and gravity waves as well as seasonal variation as we have shown in this study.

Ultimately, the $297.2 \mathrm{~nm}$ emission is directly connected to the $\mathrm{CO}_{2}$ density because the ${ }^{1} \mathrm{~S}$ excited state of atomic oxygen is only produced by $\mathrm{CO}_{2}$ photodissociation at these altitudes. Carbon dioxide is the main atmospheric component, its density and temperature are key parameters to understand the physical processes that drive the climate in the Martian environment.

The extensive study of the characteristics of the OI dayglow emission at $297.2 \mathrm{~nm}$ can be summarized to the following key conclusions:

- The altitude of the $297.2 \mathrm{~nm}$ emissions peaks varies by $\sim 20 \mathrm{~km}$ during a Martian year.

- A similar trend is observed in the altitude variation of the $\mathrm{CO}_{2}^{+}$ UV doublet emission located $\sim 5 \mathrm{~km}$ higher than the upper peak of $297.2 \mathrm{~nm}$ emission.

- The altitudes of all three emissions peaks are controlled by the overlying $\mathrm{CO}_{2}$ column density.

- Our dayglow model in combination with MCD simulations reproduces the seasonal trend of the observations.

- The best agreement with the observations is obtained if the MCD $\mathrm{CO}_{2}$ density is decreased by $40 \%$ above $\sim 80 \mathrm{~km}$ and increased by $30 \%$ above $\sim 120 \mathrm{~km}$.

\section{Acknowledgments}

This research was partly funded by the NOMAD PRODEX program managed by the European Space Agency with help of the Belgian Science Policy Office (BELSPO) and by BELSPO, Belgium's SCOOP/BRAIN research contract. B.H. is supported by the Belgian Fund for Scientific Research (FNRS). This research was supported by NASA, United States through the MAVEN project. The IUVS and EUVM MAVEN datasets were obtained from NASA's Planetary Data System (PDS) available at http://atmos.nmsu.edu/data_and_services/atmospheres_data/MAVEN/ maven_main.html. The IUVS archives are Version 13. This work utilized the RMACC Summit supercomputer, which is supported by the National Science Foundation, United States (awards ACI-1532235 and ACI-1532236), the University of Colorado Boulder, United States, and Colorado State University, United States. The Summit supercomputer is a joint effort of the University of Colorado Boulder and Colorado State University. The Mars Climate Database (version 5.3) is available online at http://www-mars.lmd.jussieu.fr/mars/access.html. We thank Millour E. for his assistance with the LMD MCD model.

\section{References}

Barth, C.A., Hord, C.W., Pearce, J.B., Kelly, K.K., Anderson, G.P., Stewart, A.I., 1971. Mariner 6 and 7 ultraviolet spectrometer experiment: Upper atmosphere data. J. Geophys. Res. 17 (10), 2213-2227, 76 Icarus.

Barth, C.A., Stewart, A.I., Hord, C.W., Lane, A.L., 1972. Mariner 9 ultraviolet spectrometer experiment: Mars airglow spectroscopy and variations in Lyman alpha. Icarus 17 (2), 457-468.

Bertaux, J.-L., et al., 2006. SPICAM on Mars express: Observing modes and overview of UV spectrometer data and scientific results. J. Geophys. Res. 111, E10S90.

Chantler, C.T., Nguyen, T.V.B., Lowe, J.A., Grant, I.P., 2013. Relativistic calculation of transition probabilities for $557.7 \mathrm{~nm}$ and $297.2 \mathrm{~nm}$ emission lines in oxygen. Astrophys. J. 769 (1), 84.

Eparvier, F.G., Chamberlin, P.C., Woods, T.N., Thiemann, E.M.B., 2015. The solar extreme ultraviolet monitor for MAVEN. Space Sci. Rev. 195 (1-4), 293-301.
Forget, F., Hourdin, F., Fournier, R., Hourdin, C., Talagrand, O., Collins, M., Huot, J.P., et al., 1999. Improved general circulation models of the Martian atmosphere from the surface to above $80 \mathrm{~km}$. J. Geophys. Res. Planets 104 (E10), 24155-24175.

Forget, F., Montmessin, F., Bertaux, J.-L., González-Galindo, F., Lebonnois, S., Reberac, A., 2009. Density and temperatures of the upper Martian atmosphere measured by stellar occultations with Mars express SPICAM. J. Geophys. Res. 114, E01004.

Fox, J.L., Dalgarno, A., 1979. Ionization, luminosity, and heating of the upper atmosphere of Mars. J. Geophys. Res. 84 (A12), 7315-7333.

Gérard, J.-C., Gkouvelis, L., Ritter, B., Hubert, B., Jain, S.K., Schneider, N.M., 2019. Maven-iuvs observations of the $\mathrm{CO}_{2}{ }^{+}$uv doublet and co cameron bands in the martian thermosphere: aeronomy, seasonal, and latitudinal distribution. Journal of Geophysical Research (Space Physics) 124 (7), 5816-5827. http://dx.doi.org/10. 1029/2019JA026596.

Gkouvelis, L., Gérard, J.-C., Ritter, B., Hubert, B., Schneider, N.M., Jain, S.K., 2018. The $\mathrm{O}\left({ }^{1} S\right)$ 297.2-nm dayglow emission: A tracer of $\mathrm{CO}_{2}$ density variations in the Martian lower thermosphere. Journal of Geophysical Research (Planets) 123, 3119-3132.

González-Galindo, F., Chaufray, J.-Y., Forget, F., Garcia-Comas, M., Montmessin, F., Jain, S.K., Stiepen, A., 2018. UV dayglow variability on Mars: Simulation with a global climate model and comparison with SPICAM/MEx data. J. Geophys. Res. Planets 123, 1934-1952.

González-Galindo, F., López-Valverde, M.A., Angelats i Coll, M., Forget, F., 2005. Extension of a martian general circulation model to thermospheric altitudes: uv heating and photochemical models. Journal of Geophysical Research (Planets) 110 (E9), E09008. http://dx.doi.org/10.1029/2004JE002312.

Gröller, H., Montmessin, F., Yelle, R.V., Lefevre, F., Forget, F., Schneider, N.M., Jain, S.K., et al., 2018. MAVEN/IUVS stellar occultation measurements of Mars atmospheric structure and composition. J. Geophys. Res. Planets 123, 1449-1483.

Hanson, W.B., Sanitani, S., Zuccaro, D.R., 1977. The Martian ionosphere as observed by the Viking retarding potential analyzers. J. Geophys. Res. 82, 4351-4363.

Hess, S.L., et al., 1980. The annual cycle of pressure on Mars measured by Viking landers 1 and 2. Geophys. Res. Lett. 7.3, 197-200.

Jain, S.K., Stewart, A.I.F., Schneider, N.M., Deighan, J., Stiepen, A., Evans, J.S., Clarke, J.T., et al., 2015. The structure and variability of Mars upper atmosphere as seen in MAVEN/IUVS dayglow observations. Geophys. Res. Lett. 42 (21), 9023-9030.

Jakosky, Bruce M., 2017. Maven observations of the mars upper atmosphere, ionosphere, and solar wind interactions. Journal of Geophysical Research (Space Physics) 122 (9), 9552-9553. http://dx.doi.org/10.1002/2017JA024324.

Kramida, A., Yu, Ralchenko, Reader, J., NIST ASD Team, 2018. NIST atomic spectra database. National Institute of Standards and Technology, Gaithersburg, MD, ver. 5.5.6. https://physics.nist.gov/asd. (Accessed 10 April 2018).

Leblanc, F., Chaufray, J.Y., Lilensten, J., Witasse, O., Bertaux, J.L., 2006. Martian dayglow as seen by the SPICAM UV spectrograph on Mars express. J. Geophys. Res. Planets 111 (E9).

McClintock, W.E., Schneider, N.M., Holsclaw, G.M., Clarke, J.T., Hoskins, A.C., Stewart, I., Deighan, J., et al., 2015. The imaging ultraviolet spectrograph (IUVS) for the MAVEN mission. Space Sci. Rev. 195 (1-4), 75-124.

Millour, E., et al., 2015. The Mars climate database (MCD version 5.2). EPSC abstract 2015-438, Vol. 10

Montabone, L., Forget, F., Millour, E., Wilson, R.J., Lewis, S.R., Cantor, B., Kass, D., et al., 2015. Eight-year climatology of dust optical depth on Mars. Icarus.

Nier, A.O., McElroy, M.B., 1977. Composition and structure of Mars' upper atmosphere: Results from the neutral mass spectrometers on Viking 1 and 2. J. Geophys. Res. $82,4341-4349$.

Stevens, M.H., Evans, J.S., Schneider, N.M., Stewart, A.I.F., Deighan, J., Jain, S.K., Holsclaw, G.M., et al., 2015. New observations of molecular nitrogen in the Martian upper atmosphere by IUVS on MAVEN. Geophys. Res. Lett. 42 (21), 9050-9056.

Stewart, A.I., 1972. Mariner 6 and 7 ultraviolet spectrometer experiment: implications of $\mathrm{CO}_{2}^{+}$, $\mathrm{CO}$ and O airglow. J. Geophys. Res. 77 (1), 54-68.

Stewart, A.I., Barth, C.A., Lane, A.L., 1972. Mariner 9 ultraviolet spectrometer experiment: Structure of Mars' upper atmosphere. Icarus 17, 469-474.

Thiemann, E., Chamberlin, P., Eparvier, F., Woods, T., Bougher, S., Jakosky, B., 2016. The MAVEN/EUVM level 3 spectral irradiance model: Algorithms and results. J. Geophys. Res. 122, 2748-2767.

Venot, O., Bénilan, Y., Fray, N., Gazeau, M.C., Lefèvre, F., Es-sebbar, E., Lefèvre, M., et al., 2018. VUV-absorption cross section of carbon dioxide from 150 to $800 \mathrm{~K}$ and applications to warm exoplanetary atmospheres. Astron. Astrophys. 609 (A34).

Withers, P., Pratt, R., 2013. An observational study of the response of the upper atmosphere of Mars to lower atmospheric dust storms. Icarus 225 (1), 378-389.

Yoshino, K., Esmond, J.R., Sun, Y., Parkinson, W.H., Ito, K., Matsui, T., 1996. Absorption cross section measurements of carbon dioxide in the wavelength region 118.7-175.5 $\mathrm{nm}$ and the temperature dependence. J. Quant. Spectrosc. Radiat. Transfer 55 (1), 53-60. 